\title{
Multiband Antenna for Portable Device Applications
}

\author{
Mohammad Amin Honarvar, Naeemehsadat Hamidi, Bal Singh Virdee
}

\begin{abstract}
- this paper describes a compact planar microstrip antenna for multiband transceiver application. The proposed antenna consists of a combination of E-shaped and C-shaped radiators that are excited via a common microstrip feed-line. Embedded in the ground-plane are included an open- and short-ended slots whose purpose is to enhance the antenna's impedance match and increase the number of bands in which the antenna can operate. A prototype antenna was fabricated on a standard FR4 substrate with $\varepsilon_{r}$ of 4.4 and thickness of 1.6 $\mathrm{mm}$, and its reflection-coefficient and radiation patterns were measured to demonstrate its feasibility. Measurements confirm the antenna can simultaneously operate over the frequency bands of: DCS (1.71-1.85 GHz), PCS (1.85-1.99 GHz), UMTS (1.92-2.17 GHz), Wibro (2.3-2.39 GHz), WLAN+ Bluetooth (2.4-2.48 MHz), WIMAX (2.5-2.69 GHz), WIMAX (3.3-3.5 GHz), HIPERLAN2 (5.15-5.35/5.47-5.725 GHz), and WLAN (5.15-5.35/5.725-5.825 GHz). The antenna has dimensions of $30 \times 40 \times 1.6 \mathrm{~mm}^{3}$, which is compatible with the requirements imposed by portable wireless systems.
\end{abstract}

Index Terms - Mobile phone, monopole antenna, broadband antenna, multiband antenna, portable devices

\section{INTRODUCTION}

One of the essential components in any wireless communication system is the antenna whose function is to interface with the propagating medium. Various wireless applications demand antennas to be compact in size and be easily integrated into the RF front-end of the system. In addition, they need to support different communications standards for various applications. A great deal of work has been carried out to date in the design of antennas for multiband communications. Various antennas have been proposed to date which include a planar inverted F-antenna and slot antenna [1-3] to support a WLAN, WiMAX, and UMTS standards. However, these antennas inherently possess a narrow bandwidth which restricts their application for portable transceivers requiring multiband operation. Several techniques have been used to improve the bandwidth of such antennas. This includes the introduction of various resonant

M.A. Honarvar is with the Electrical Engineering Department, Islamic Azad University of Najafabad, Isfahan, Iran. (e-mail: amin.honarvar@gmail.com). N. Hamidi is with the Electrical Engineering Department, Islamic Azad University of Jahrom, Fars, Iran, (e-mail:naeemeh.hamidi@gmail.com) B.S. Virdee is with London Metropolitan University, London, UK (e-mail: b.virdee@londonmet.ac.uk) elements in order to create a multiband is a very common approach [4]. Another method calls for the addition of parasitic patches with resonant lengths close to the frequency band where the bandwidth improvement is required [5]. Other techniques use multilayers of resonators in order to increase the number of bands [6]. The large size of these antennas makes them unsuitable for certain mobile applications. Recently, a compact triple-band microstrip-fed planar monopole antenna with dimension of $15 \times 15 \times 1.6 \mathrm{~mm}^{3}$ was reported [7]. Although the antenna is a compact structure, it only covers two bands, i.e. WLAN and WiMAX bands.

In this paper, a compact planar antenna is proposed which operates across several communications bands, namely, DCS/PCS/UMTS, WiBRO, WLAN, WiMAX, HIPERLAN/ IEEE 802.11a. The proposed antenna consists of E-shape and $\mathrm{C}$-shaped radiators which are excited using a microstrip feedline. Additional resonant modes are generated by including a horizontal open-circuit slot and vertical short-circuit slot on the ground-plane. The antenna provides an average gain of 2 $\mathrm{dBi}$ over a frequency range of across $1.75-5.5 \mathrm{GHz}$, and it predominately radiates omnidirectionally.

\section{ANTENNA STRUCTURE}

The configuration of proposed antenna, shown in Fig. 1, comprises of an E-shaped and $\mathrm{C}$-shaped radiators that are excited through the main microstrip feed-line. The lower arm of the E-shaped radiator is grounded through a via-hole. The ground-plane includes a wide open-circuit slot along with a short circuit slot to enhance the antenna's impedance match and generate additional resonant modes. The antenna is constructed on the FR4 substrate with permittivity of 4.4 and thickness of 1.6. The antenna occupies an area of $30 \mathrm{~mm} \times 40$ $\mathrm{mm}$. The parameters defining the antenna are: $\mathrm{Lm}=26 \mathrm{~mm}$, $\mathrm{Lp}=19 \mathrm{~mm}, \mathrm{~L} 2=7.6 \mathrm{~mm}, \mathrm{~L} 3=7 \mathrm{~mm}, \mathrm{~W} 2=10 \mathrm{~mm}, \mathrm{~W} 1=9$ $\mathrm{mm}, \mathrm{Wop}=4.35 \mathrm{~mm}, \mathrm{Lsc}=9 \mathrm{~mm}, \mathrm{Wsc}=3.3 \mathrm{~mm}$.

Insight on the antenna was achieved by establishing the distribution of current on its surface at various frequencies of interest as shown in Fig. 2. The current density distribution highlighted regions of the antenna geometry that have great influence on its performance. The surface current at the lower resonant frequency of $1.73 \mathrm{GHz}$, shown in Fig. 2(a), indicates regions of $\mathrm{C}$-shaped and E-shaped radiators that have the greatest effect at that frequency. This is essential horizontal and vertical sections. At this resonant frequency the effective length of radiators corresponds to a quarter of guided wavelength, i.e. $2 \times \mathrm{W} 2+\mathrm{L} 2=27.6 \mathrm{~mm}$ ) at $1.73 \mathrm{GHz}$. At the resonant frequency of $2.33 \mathrm{GHz}$, the coupling between the $\mathrm{C}$ - 
shaped radiator and narrow slot on the ground-plane generate a resonant frequency corresponding to an effective length equivalent to $\mathrm{Lsc}+\mathrm{W} 2=19 \mathrm{~mm}$. At $3.5 \mathrm{GHz}$ the surface current density is concentrated in the lower region of the Eshaped radiator, which couples electromagnetic energy to the right side of ground slot through the via. Finally, at $5.5 \mathrm{GHz}$ the radiation is mainly dissipated from the open-ended stub and the narrow slot. The effective length of the current path is equal to Lsc $=9 \mathrm{~mm}$ which is approximately a quarter of the guided wavelength at $5.5 \mathrm{GHz}$.

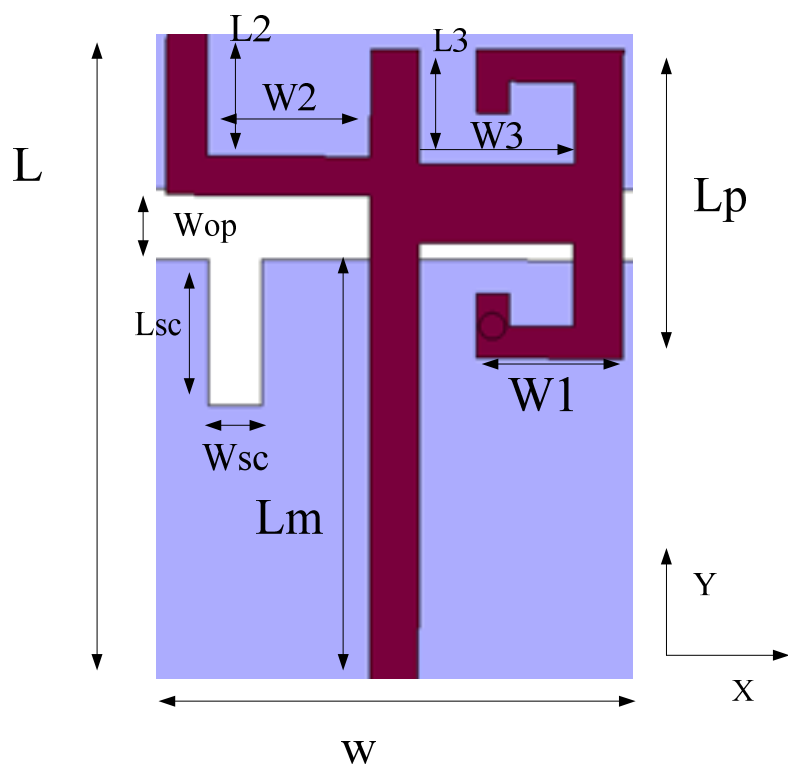

Fig. 1. Geometry of the proposed antenna.

\section{RESUlTS AND Discussion}

The proposed antenna was fabricated on dielectric substrate FR4 with dielectric constant $=4.4$ and thickness $=1.6 \mathrm{~mm}$. It has dimensions of $30 \times 40 \times 1.6 \mathrm{~mm}^{3}$. The photograph of the constructed antenna is shown in Fig. 3. The simulated and measured reflection-coefficient of the antenna is shown in Fig. 4. The antenna operates over the frequency ranges of 1.73$2.97 \mathrm{GHz}$ to cover DCS, PCS, UMTs, WiBro systems; and 2.4-2.8 GHz, 3.31-3.68 GHz, 5.03-5.93 GHz to cover the bands of WLAN+ Bluetooth/ WIMAX/ HIPERLAN2/ IEEE 802.11 a systems, respectively.

The radiation patterns of proposed antenna in the E- and Hplanes ( $x z$-plane and $y z$-plane) at $1.75 \mathrm{GHz}, 3.5 \mathrm{GHz}$ and 5.5 $\mathrm{GHz}$ are shown in Fig. 5. These results confirm that antenna predominately radiates energy omnidirectionally. The antenna exhibits a dipole-like property in the low frequency band. At the high frequency bands it exhibit approximately omnidirectional characteristics. The measured maximum gain of antenna is shown in Fig. 6. The gain varies between $0.5 \mathrm{dBi}$ at $1.75 \mathrm{GHz}$ to $3 \mathrm{dBi}$ at $5.5 \mathrm{GHz}$. The antenna performance confirms its viability for commercial portable wireless systems.

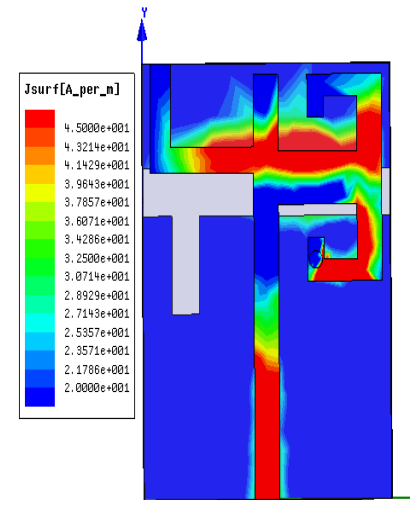

(a)

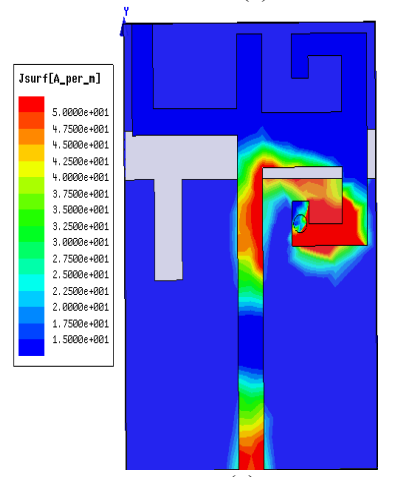

(c)

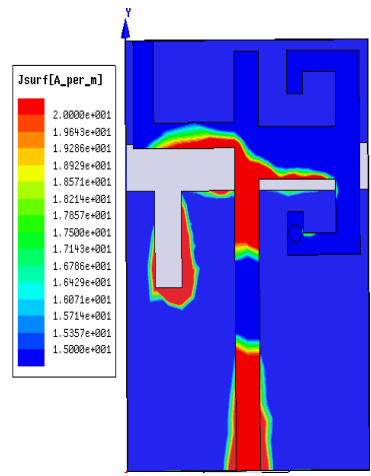

(b)

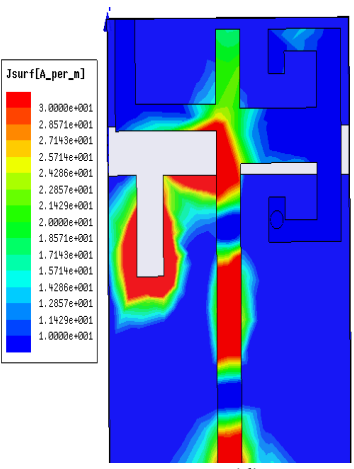

(d)
Fig. 2. The computed surface current density of the proposed antenna at: (a) $1.73 \mathrm{GHz}$, (b) $2.33 \mathrm{GHz}$, (c) $3.5 \mathrm{GHz}$ and, (d) $5.5 \mathrm{GHz}$.

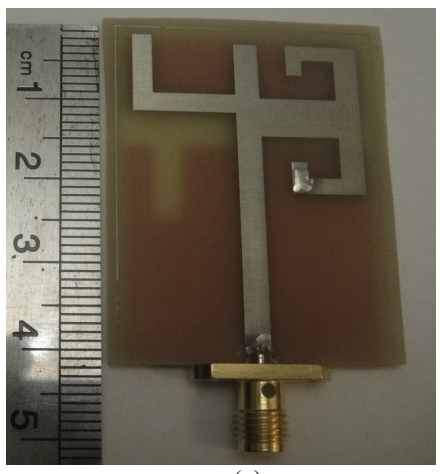

(a)

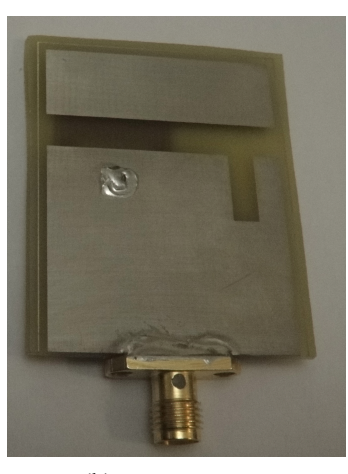

(b)
Fig. 3. Photograph of the fabricated antenna, (a) top layer, and (b) bottom layer.

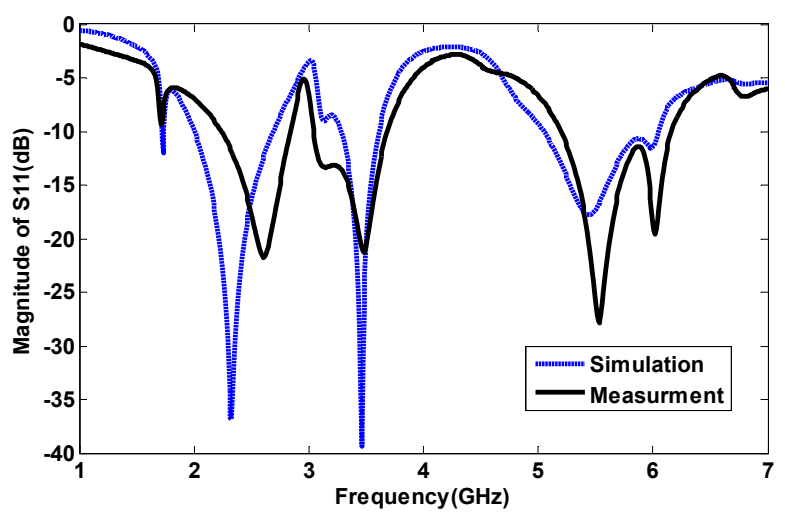

Fig. 4. The simulated and measured reflection-coefficient of the antenna. 


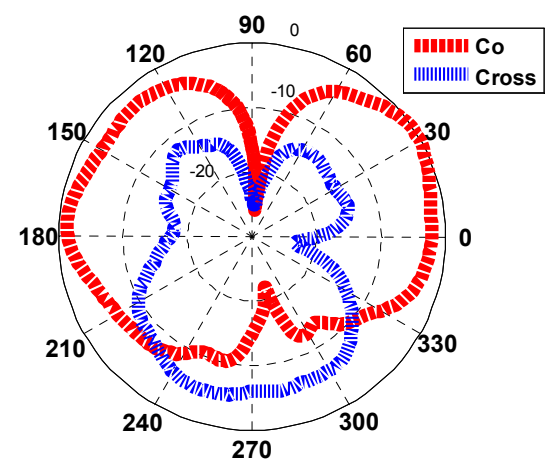

(a)

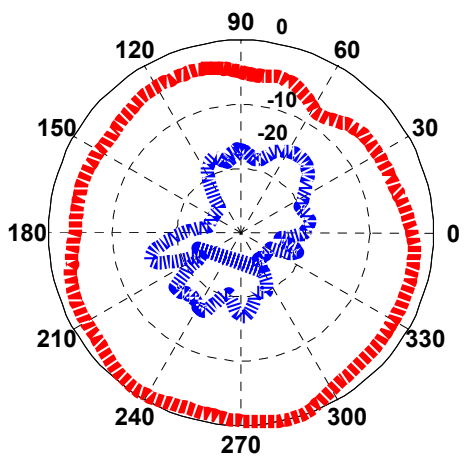

(b)

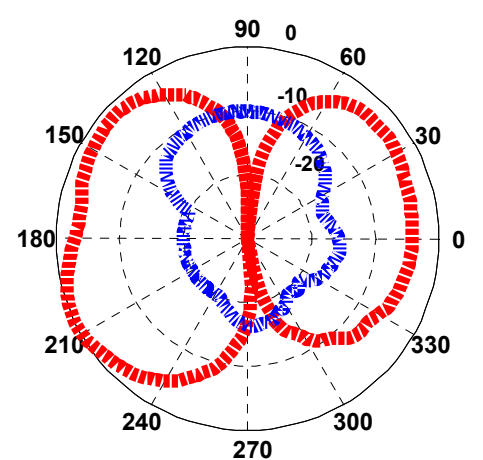

(c)

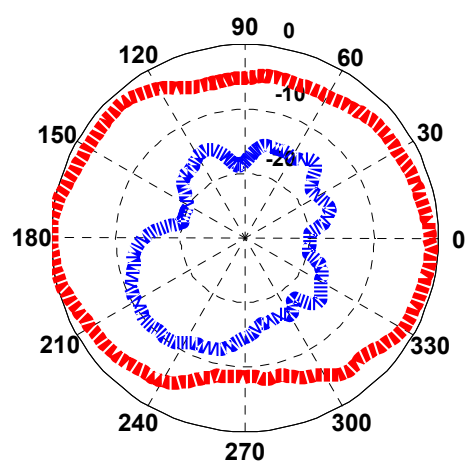

(d)

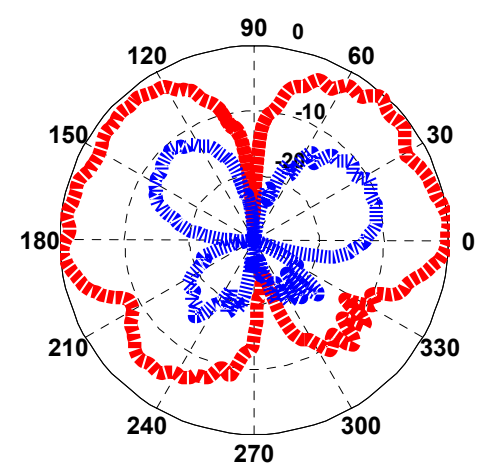

(e)

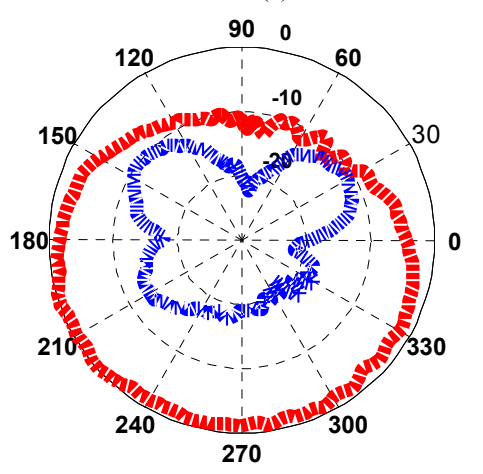

(f)

Fig. 5. The measured radiation pattern of the antenna: (a) xz-plane at 1.73 $\mathrm{GHz}$, (b) yz-plane at $1.73 \mathrm{GHz}$, (c) xz-plane at $3.5 \mathrm{GHz}$, (d) yz-plane at 3.5 $\mathrm{GHz},(\mathrm{e}) \mathrm{xz}$-plane at $5.5 \mathrm{GHz}$, and (f) yz-plane at $5.5 \mathrm{GHz}$.

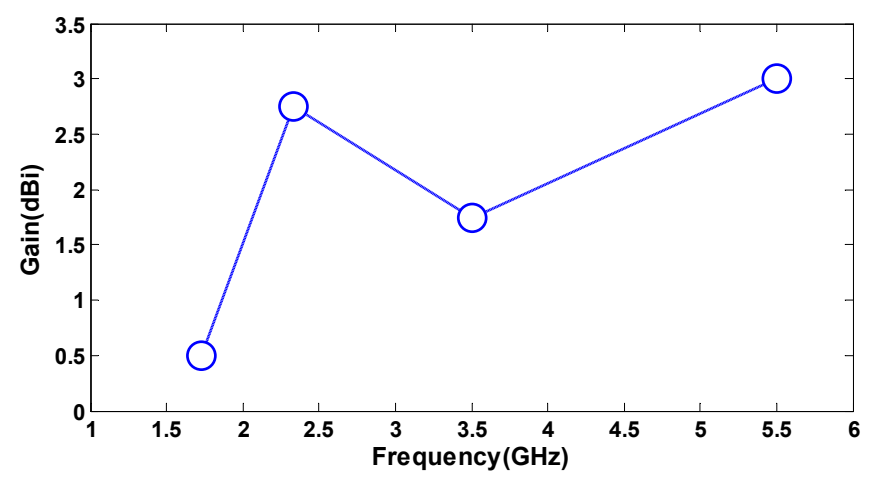

Fig. 6. The measured gain of the proposed antenna.

\section{CONCLUSION}

A technique is described to implement a multiband antenna for portable wireless communications systems. The antenna structure consists of E-shaped and C-shaped radiators that are excited through a common microstrip feed-line. An opencircuit slot and narrow short-circuit slot are embedded in the ground-plane of the antenna to improve its impedance matching and generate additional resonant frequencies. The measured results confirm the antenna can simultaneously support various communications bands, namely DCS/PCS/UMTS/WiBro/WLAN and Bluetooth/WLAN/ WIMAX/HIPERLAN2/IEEE 802.11a. The antenna possesses 
omnidirectional radiation characteristics across the specified bands, and provides gain an average gain of $2 \mathrm{dBi}$ across $1.75-$ 5.5 GHz. Easy construction without a matching network or a complicated geometry is an additional feature of the antenna that reflects on its low fabrication cost. In addition, the antenna is compact in size with dimension of $30 \times 40 \times 1.6 \mathrm{~mm}^{3}$.

\section{REFERENCES}

[1] P. Ciais, R. Staraj, G. Kossiavas, and C. Luxey, "Compact Internal Multiband Antenna for Mobile Phone and WLAN Standards," Electronic Letters. vol. 40, no.15, pp.920921, July 2004.

[2] J. Anguera, I. Sanz, J. Mumbru, and C. Puenta, "Multiband and Set Antenna With a Parallel Excitation of PIFA and Slot Radiators," IEEE Trans. Antennas Propag., vol. 58, no. 2, pp. 348-356, Feb. 2010.

[3] J.-H. Kim, Y.-B. Chae, J.-H. Lim, T.-Y. Yun, "Printed internal antenna for mobile broadcasting (DVB-H/TDMB) and communications (GSM900)," IET Microwaves, Antennas \& Propagation, vol. 6, no. 6, pp. 680-684, 2012.

[4] Bhatti, R.A., Y. Im, and S. Park, "Compact PIFA for mobile terminals supporting cellular and non-cellular standards," IEEE Transactions on Antennas and Propagation, vol. 57, 2534-2540, 2009.

[5] Cho, Y. J., S.H. Hwang, and S.O. Park, "A dual-band internal antenna with a parasitic patch for mobile handsets and the consideration of the handset case and battery," IEEE Antennas and Wireless Propagation Letters, vol. 4, 429-432, 2005.

[6] Bhatti, R. A., Y. Im, and S. Park, "Compact PIFA for mobile terminals supporting cellular and non-cellular standards," IEEE Transactions on Antennas and Propagation, Vol. 57, 2534-2540, 2009.

[7] M. Moosazadeh, S. Kharkovsky, "Compact and small planar monopole antenna with symmetrical L-and Ushaped slots for WLAN/WiMAX Applications," IEEE Antenna and Wireless propag. Lett., vol. 13, pp. 388-391, 2014. 